\title{
Characterizing the metabolites related to rice salt tolerance with introgression lines exhibiting contrasting performances in response to saline conditions
}

\author{
Ziyan Xie $^{1} \cdot$ Chunchao Wang ${ }^{1} \cdot$ Shuangbing $Z^{2} u^{2} \cdot$ Wensheng Wang ${ }^{1} \cdot$ Jianlong Xu ${ }^{1} \cdot$ Xiuqin Zhao ${ }^{1}$
}

Received: 25 September 2019 / Accepted: 16 May 2020 / Published online: 6 June 2020

(c) The Author(s) 2020

\begin{abstract}
Rice is susceptible to salt stress at the seedling stage. To explore the molecular mechanisms underlying salt tolerance, the metabolic responses to salt stress were investigated with a metabolite-profiling technique. Gas chromatography-mass spectrometry was used to profile metabolite changes in five rice lines with a similar genetic background, but with obviously diverse growth performances under saline conditions. A total of 84 metabolites were detected in rice leaf extracts under control and saline conditions. The data revealed that amino acids were enriched more in three salt-tolerant lines (G58, G1710, and IR64) than in two salt-sensitive lines (G45 and G52) under control conditions, suggesting that there were basal metabolite differences between the tolerant and sensitive lines. Additionally, significantly higher allantoin levels in G58, G1710, and IR64 under both stress and control conditions were observed, implying allantoin was important for the better growth of the three rice lines. Moreover, sorbitol, melezitose, and pipecolic acid levels increased considerably in response to salt stress in the five lines, indicating they contribute to rice responses to salt stress significantly. Interestingly, the similar metabolic patterns were regulated by salt stress in the salt-sensitive and salt-tolerant lines, and the main difference was quantitative. The sensitive lines had more pronounced increases during the early stages of the stress treatment than the tolerant lines. Thus, monitoring the metabolome changes of plants may provide crucial insights into how plants tolerate stress. The results presented herein provide valuable information for further elucidating the molecular mechanisms underlying rice salt tolerance.
\end{abstract}

Keywords Rice $\cdot$ Metabolites $\cdot$ Salt tolerance $\cdot$ Introgression lines

\section{Introduction}

Salt stress is a major constraint in many rice-producing regions because modern rice varieties are highly sensitive to this abiotic stress especially at the seedling and flowering

Electronic supplementary material The online version of this article (https://doi.org/10.1007/s10725-020-00627-y) contains supplementary material, which is available to authorized users.

Jianlong Xu

xujianlong@caas.cn

$\triangle$ Xiuqin Zhao

zhaoxiuqin@caas.cn

1 Institute of Crop Sciences/National Key Facility for Crop Gene Resources and Genetic Improvement, Chinese Academy of Agricultural Sciences, Beijing 100081, China

2 College of Agriculture, Yangtze University, Jingzhou 434025, China stage (Jahan et al. 2020; Singh et al. 2017; Thomson et al. 2010; Pearson and Bernstein 1959; Pearson et al. 1966). More than 800 million hectares of land are affected by high salinity worldwide (Liu et al. 2017; Munns and Tester 2008). Because of land scarcity and high food demand, marginal lands, such as saline-prone areas, must be used for agricultural production (Islam et al. 2012). Thus, the genetic improvement of salt-tolerant rice varieties appears to be the most feasible and promising strategy for maintaining stable global food production (Walia et al. 2005; Banerjee et al. 2019). How plants perceive the specific salt stress signal and transmit information internally is an important issue to improve crop productivity under stress (Liu et al. 2017).

Plants under saline conditions are exposed to hyperosmotic and hyperionic stresses, resulting in considerable productivity losses (Singh et al. 2017; Anwar et al. 2016). This ion-specific toxicity results mostly from altered ratios of potassium and sodium ions $\left(\mathrm{K}^{+} / \mathrm{Na}^{+}\right)$and/or $\mathrm{Na}^{+}$and chloride $\left(\mathrm{Cl}^{-}\right)$concentrations that adversely affect cellular 
functions (Negrão et al. 2011; Anwar et al. 2016). One of the essential mechanisms underlying salt tolerance in plants involves decreasing the effects of ionic stress by minimizing the $\mathrm{Na}^{+}$accumulation in the cytosol, particularly in the transpiring leaves (Sharma et al. 2016). Regarding hyperosmotic stress, increases in the intracellular concentration of diverse soluble organic compounds can prevent osmotic shrinkage or swelling (Widodo et al. 2009; El-Shintinawy and El-Shourbagy 2001). Therefore, avoiding ion accumulation and osmotic stress are important features of salt stress tolerance.

Plant adaptations to salt stress require a complex physiological process accompanied by changes at metabolite level (Banerjee et al. 2019). The cellular metabolite contents are usually considered a better indicator of the physiological state of cells than the transcript and protein contents (Kim et al. 2007; Anwar et al. 2016; Nemat Alla et al. 2012) because they are the result of interactions among various genes and pathways, resulting in systemic effects in response to stress (Garg et al. 2002; Taji et al. 2002; Nuccio et al. 2015). There has recently been increasing interest in metabolic responses to abiotic stress in plants because metabolome profiles are very sensitive to abiotic stresses (Okazaki and Saito 2016; Raval et al. 2018). Many metabolites are considered crucial for stress tolerance (Garg et al 2002; Nuccio et al. 2015; Ma et al. 2016) because they contribute to osmotic regulation and/or serve as free radical scavengers (Sharma et al. 2016). For example, previous studies confirmed that amino acids (AAs) and sugars accumulate in plant systems in response to salinity stress (Zhao et al. 2014; Gong et al. 2005). Mannitol reportedly serves as both a free radical scavenger and an osmotic regulator (Sharma et al. 2016). Putrescine is a polyamine that is important for maintaining plant growth and development under stress conditions (Singh et al. 2017; Pal et al. 2015; Raval et al. 2018). Increases in polyamine contents are correlated with stress tolerance in plants (Groppa and Benavides 2008; Sharma et al. 2016; Anwar et al. 2016). Therefore, monitoring the metabolome changes of plants may provide crucial insights into how plants tolerate stress. However, compared with the extensive research on the salt tolerance-related genes/proteins in rice, there have been few investigations regarding the metabolites involved in salt tolerance.

The existing comparative metabolomics studies on rice salt tolerance mainly focused on comparisons of varieties with substantial differences in their genetic backgrounds (Widodo et al. 2009). Comparisons among introgression lines with different stress-tolerant performance but similar genetic background could decrease the influence of the genetic background on the result interpretations ( $\mathrm{Li}$ et al. 2005). Furthermore, rice were generally analyzed at 7 or more days after salt treatments when plants were exhibiting obvious stress-induced symptoms (Zuther et al. 2007), which has resulted in relatively little information regarding the early metabolic responses. Therefore, in the present study, we analyzed the metabolic changes in rice plants in response to salinity stress at 3 days after treatments (i.e., almost no stress-induced symptoms), with four introgression lines with the same genetic background and the recurrent parent IR64 as research materials. We identified 84 metabolites based on gas chromatography-mass spectrometry (GC-MS) technology, and observed that the abundance of most metabolites significantly increased under saline conditions. Differences in the metabolite levels between lines with diverse responses to salinity stress are discussed herein. The results of this study may extend our understanding of the metabolic mechanism underlying salt tolerance in rice.

\section{Materials and methods}

Five lines with a similar genetic background (G45, G52, G58, G1710, and IR64) were used as experimental materials. Lines $\mathrm{G} 45$, G52, G58, and $\mathrm{G} 1710$ are $\mathrm{BC}_{2} \mathrm{~F}_{8}$ introgression lines with the IR64 genetic background, and were derived from a cross between IR64 (indica, International Rice Research Institute) and Tarom molaii (Japonica, Iran). Analyses over successive years revealed that among the analyzed lines, G45 and G52 are the most adversely affected by salt stress, whereas G1710 is the most tolerant. IR64 is a popular commercial variety exhibiting moderate salt tolerance; Tarom molaii, a landrace introduced from Iran, is grown under irrigated temperate conditions alongside the Caspian Sea coastal belt of northern Iran. The salt occurs by saline sea water intrusions must have allowed favorable genes for salt tolerance to get accumulated in this landrace over centuries. As our previous reports, although the donor parent Tarom molaii is not ideal for salt tolerance under the experimental condition, it does possess favorable alleles for salt tolerance as compared with modern variety IR64 (Sun et al. 2007).

\section{Plant growth conditions}

A pot experiment was designed to compare the responses of the rice lines to salt stress which conducted in the glasshouse at Institute of Crop Sciences, the Chinese Academy of Agricultural Sciences (CAAS) in Beijing. The greenhouse conditions were set as $30-20{ }^{\circ} \mathrm{C}$ (day-night) and 60-65\% relative humidity were regulated with a roof net, ventilation system and cooling pad. Rice plants were cultivated in nutrient solution (Yoshida et al. 1976). The rice were treated with $100 \mathrm{mM} \mathrm{NaCl}$ for an electrical conductivity of $12 \mathrm{dS} \mathrm{m}^{-1}$ as salt treatment when seedling have three complete leaves and the fourth leaf just come out. The electrical conductivity of the control treatment was approximately $1.0 \mathrm{dS} \mathrm{m}^{-1}$. Both 
control and salt stress treatments were completed with five replicates.

\section{Metabolite extraction and identification}

The top-most leaves of plants cultivated under control and stress conditions were simultaneously collected at 3 days after salt treatments. The samples were flash-frozen in liquid nitrogen and stored at $-70{ }^{\circ} \mathrm{C}$ for a subsequent metabolite extraction and identification, which was completed as reported (Zhao et al. 2014, 2018; Bowne et al. 2012).

\section{Shoot and root $\mathrm{Na}^{+}$and $\mathrm{K}^{+}$contents}

The shoot and root $\mathrm{Na}^{+}$and $\mathrm{K}^{+}$contents at 8 days after salt treatments were estimated with a flame photometer (S2; Thermo Finnigan, Waltham, MA, USA) as previously described (Zhao et al. 2014).

\section{Growth measurements}

Rice plants were harvested at 8 days after salt treatments. Shoot height (SHT), root length (RL, axial root length), and dry weights of shoot and root (SDW and RDW) were measured for salt-stressed and control plants.

\section{Data analysis}

Datasets underwent a one-way analysis of variance (ANOVA) with the SAS 8 software (SAS Institute Inc. Cary, NC, USA). Duncan's multiple range tests were conducted to evaluate the significance of the differences in mean values among treatments. The means and standard errors for all analyzed growth and physiological parameters and metabolite contents were calculated based on five replicates. Significant differences in the traits or metabolite concentrations among the analyzed lines were determined at $\mathrm{P} \leq 0.05$. The normalized metabolite data were further subjected to a principal component analysis with the SPSS13.0 software (https://www.ibm.com/products/spss-statistics).

\section{Results}

\section{General growth performance of hydroponically grown rice plants}

Under salt stress conditions, G58, G1710, and IR64 grew better than G45 and G52. Specifically, the SHT, SDW, and RDW were generally greater for G1710, G58, and IR64 than for G45 and G52 (Table 1). For example, the SHT (SDW) was $36.8 \mathrm{~cm}\left(110.6 \mathrm{mg} \cdot\right.$ plant $\left.^{-1}\right), 35.0 \mathrm{~cm}\left(93.3 \mathrm{mg} \cdot\right.$ plant $\left.^{-1}\right)$, $33.0 \mathrm{~cm}\left(83.8 \mathrm{mg} \cdot \mathrm{plant}^{-1}\right), 30.1 \mathrm{~cm}\left(53.8 \mathrm{mg} \cdot\right.$ plant $\left.^{-1}\right)$, and $32.2 \mathrm{~cm}\left(48.1 \mathrm{mg} \cdot\right.$ plant $\left.^{-1}\right)$ for G1710, G58, IR64, G45, and G52, respectively. An analysis of plants grown under control conditions revealed the SDW and RDW were significantly higher for G58, G1710, and IR64 than for G45 and G52.

Compared with the effects of the control treatment, the salt treatment considerably decreased shoot growth (Table 1). The SHT (SDW) decreased by $4.2 \%$ (13.8\%), $6.2 \%(26.3 \%), 8.3 \%$ (35.9\%), 9.6\% (40.8\%), and $10.8 \%$ (49.8\%) for G1710, G58, IR64, G45, and G52, respectively. The growth of G58, IR64, and especially G1710 was less adversely affected by salt stress than the growth of G45 and G52.

\section{The contents of sodium and potassium in shoot and root}

Under salt stress, the $\mathrm{Na}^{+}$concentration in shoot and root were significantly lower for G58, G1710, and IR64 than for G45 and G52 (Table 2). For example, the $\mathrm{Na}^{+}$concentrations in shoot (root) were 19.4 (14.6) $\mathrm{g} \cdot \mathrm{kg}^{-1}, 19.9$ (10.2) $\mathrm{g} \cdot \mathrm{kg}^{-1}, 19.1(14.5) \mathrm{g} \cdot \mathrm{kg}^{-1}, 27.1(19.1) \mathrm{g} \cdot \mathrm{kg}^{-1}$, and 26.3(21.8) $\mathrm{g} \cdot \mathrm{kg}^{-1}$ for G1710, G58, IR64, G45, and G52, respectively. Moreover, the $\mathrm{Na}^{+} / \mathrm{K}^{+}$ratios for the shoots (roots) were 0.96
Table 1 The rice growth parameters under both control (C) and stress (S) conditions

\begin{tabular}{|c|c|c|c|c|c|c|}
\hline & & G45 & G52 & G58 & G1710 & IR64 \\
\hline SHT & $\mathrm{C}$ & $33.3 \pm 1.0 \mathrm{~b}$ & $36.1 \pm 0.6 \mathrm{a}$ & $37.3 \pm 1.1 \mathrm{a}$ & $38.4 \pm 1.3 \mathrm{a}$ & $36.0 \pm 1.6 \mathrm{a}$ \\
\hline$(\mathrm{cm})$ & $\mathrm{S}$ & $30.1 \pm 0.7 \mathrm{~d}^{*}$ & $32.2 \pm 0.6 \mathrm{~d}^{*}$ & $35.0 \pm 0.8 b^{*}$ & $36.8 \pm 0.8 \mathrm{a}$ & $33.0 \pm 0.5 \mathrm{c}^{*}$ \\
\hline RL & $\mathrm{C}$ & $10.22 \pm 0.92$ & $9.20 \pm 0.69$ & $10.72 \pm 0.82$ & $9.38 \pm 0.35$ & $10.73 \pm 0.43$ \\
\hline$(\mathrm{cm})$ & $\mathrm{S}$ & $9.34 \pm 0.22$ & $8.76 \pm 0.47$ & $10.28 \pm 0.62$ & $9.02 \pm 0.65$ & $10.12 \pm 0.66$ \\
\hline SDW & $\mathrm{C}$ & $90.9 \pm 8.4 b$ & $95.8 \pm 4.3 \mathrm{~b}$ & $126.5 \pm 9.6 \mathrm{a}$ & $128.3 \pm 6.5 \mathrm{a}$ & $130.8 \pm 6.1 \mathrm{a}$ \\
\hline (mg/plant) & $\mathrm{S}$ & $53.8 \pm 1.6 \mathrm{c}^{*}$ & $48.1 \pm 4.0 \mathrm{~d}^{*}$ & $93.3 \pm 9.8 b^{*}$ & $110.6 \pm 5.8 \mathrm{a}$ & $83.8 \pm 7.2 b^{*}$ \\
\hline RDW & $\mathrm{C}$ & $9.23 \pm 0.44 c$ & $7.70 \pm 0.80 \mathrm{~d}$ & $10.97 \pm 0.40 \mathrm{ab}$ & $10.20 \pm 0.26 b$ & $11.22 \pm 0.27 \mathrm{a}$ \\
\hline (mg/plant) & $\mathrm{S}$ & $8.04 \pm 0.19 \mathrm{c}^{*}$ & $7.05 \pm 0.50 \mathrm{~d}$ & $10.27 \pm 0.71 \mathrm{ab}$ & $9.83 \pm 0.34 \mathrm{a}$ & $10.56 \pm 0.36 b$ \\
\hline
\end{tabular}

The alphabets "a-d" indicates the metabolites levels in five rice lines were significantly different under same one condition

*Indicates the metabolites levels changed significantly under between stress and control conditions 
Table 2 The contents of $\mathrm{Na}^{+}(\mathrm{g} /$ $\mathrm{kg})$ and $\mathrm{K}^{+}(\mathrm{g} / \mathrm{kg})$ in shoot and root of rice under control and stress conditions, respectively

\begin{tabular}{llllllll}
\hline & Tissue & Ions & G45 & G52 & G58 & G1710 & IR64 \\
\hline Stress & Shoot & $\mathrm{Na}$ & $27.06 \pm 1.55 \mathrm{a}^{*}$ & $26.27 \pm 1.24 \mathrm{a}^{*}$ & $19.95 \pm 1.07 \mathrm{~b}^{*}$ & $19.38 \pm 1.12 \mathrm{~b}^{*}$ & $19.11 \pm 2.05 \mathrm{~b}^{*}$ \\
& & $\mathrm{~K}$ & $20.5 \pm 1.5$ & $20.1 \pm 1.4$ & $19.9 \pm 3.8$ & $20.2 \pm 2.1$ & $18.5 \pm 1.4^{*}$ \\
& & $\mathrm{Na} / \mathrm{K}$ & 1.32 & 1.31 & 1.00 & 0.96 & 1.03 \\
& \multirow{2}{*}{ Root } & $\mathrm{Na}$ & $19.07 \pm 1.40 \mathrm{a}^{*}$ & $21.82 \pm 1.67 \mathrm{a}^{*}$ & $10.18 \pm 0.00 \mathrm{c}^{*}$ & $14.64 \pm 0.69 \mathrm{~b}^{*}$ & $14.52 \pm 1.11 \mathrm{~b}^{*}$ \\
& $\mathrm{~K}$ & $12.5 \pm 1.9 \mathrm{a}^{*}$ & $14.8 \pm 1.4 \mathrm{a}^{*}$ & $8.8 \pm 0.1 \mathrm{c}^{*}$ & $10.6 \pm 0.9 \mathrm{~b}^{*}$ & $12.1 \pm 0.6 \mathrm{a}^{*}$ \\
& & $\mathrm{Na} / \mathrm{K}$ & 1.53 & 1.47 & 1.16 & 1.38 & 1.20 \\
Control & Shoot & $\mathrm{Na}$ & $0.58 \pm 0.02 \mathrm{~b}$ & $0.67 \pm 0.10 \mathrm{ab}$ & $0.52 \pm 0.08 \mathrm{~b}$ & $0.81 \pm 0.03 \mathrm{a}$ & $0.41 \pm 0.05 \mathrm{c}$ \\
& $\mathrm{K}$ & $23.0 \pm 1.4 \mathrm{ab}$ & $21.6 \pm 0.7 \mathrm{bc}$ & $23.2 \pm 1.7 \mathrm{ab}$ & $19.6 \pm 1.3 \mathrm{c}$ & $26.0 \pm 1.8 \mathrm{a}$ \\
& & $\mathrm{Na} / \mathrm{K}$ & 0.03 & 0.03 & 0.02 & 0.04 & 0.02 \\
& $\mathrm{Na}$ & $1.38 \pm 0.07 \mathrm{a}$ & $0.96 \pm 0.14 \mathrm{~b}$ & $0.98 \pm 0.03 \mathrm{~b}$ & $1.23 \pm 0.10 \mathrm{a}$ & $1.08 \pm 0.01 \mathrm{~b}$ \\
& $\mathrm{~K}$ & $20.3 \pm 2.2 \mathrm{a}$ & $22.2 \pm 2.7 \mathrm{a}$ & $11.7 \pm 0.1 \mathrm{c}$ & $14.6 \pm 0.5 \mathrm{~b}$ & $14.4 \pm 1.2 \mathrm{~b}$ \\
& $\mathrm{Na} / \mathrm{K}$ & 0.07 & 0.04 & 0.08 & 0.08 & 0.08 \\
\hline
\end{tabular}

The alphabets " $\mathrm{a}-\mathrm{d}$ " indicates the metabolites levels in five rice lines were significantly different under same one condition

*Indicates the metabolites levels changed significantly under between stress and control conditions
(1.38), 1.0 (1.16), 1.03 (1.20), 1.30 (1.47), and 1.32 (1.53) for G1710, G58, IR64, G45, and G52, respectively. Of the five lines, G1710 had the lowest shoot $\mathrm{Na}^{+} / \mathrm{K}^{+}$ratio.

Compared with the control plants, the salt-stressed plants had significantly higher $\mathrm{Na}^{+}$concentrations in shoot and root (Table 2). Specifically, the salt treatment caused the leaf (root) $\mathrm{Na}^{+}$contents to increase by 22.9 (10.9), 37.4 (9.4), 45.6 (12.4), 38.2 (21.7), and 45.7 (12.8) fold in G1710, G58, IR64, G52, and G45, respectively. Moreover, the leaf (root) $\mathrm{Na}^{+} / \mathrm{K}^{+}$ratios increased by $22.2(10.9), 43.7$ (12.8), 64.5 (15.0), 41.0 (33.1), and 51.3 (21.4) fold in G1710, G58, IR64, G52, and G45, respectively. Thus, the increase in the leaf $\mathrm{Na}^{+}$content and $\mathrm{Na}^{+} / \mathrm{K}^{+}$ratio was lowest in G1710.

\section{Overview of the metabolites in leaves under saline and control conditions}

Totally 84 metabolites were identified with metabolic profiling technology which included 19 amino acids (AA), 23 sugars, 24 organic acids (OA) and 18 other small molecular components (SMC) (Table 3). A multivariate principal component analysis was used to separate biological samples based on the contribution of a set of metabolites. Figure 1 provides an overview of the changes in the metabolite pools of the leaves of rice plants acclimating to salt stress. The two principal components clearly separated the treatments and lines with different growth performances (Fig. 1). The leaf samples of the control and salt-treated plants were clearly separated by $\mathrm{PC} 1$, which represented $76.6 \%$ of the variation among samples. The loading analysis revealed that the abundance of most metabolites significantly increased in response to salt stress (Fig. 1; Supplementary Table 1). Additionally, PC2 distinguished G48 and G52 from G58, G1710, and IR64, and explained $7.7 \%$ of the variation among samples
(Fig. 1). The loading analysis indicated four OAs (i.e., citric acid, phosphate, gluconic acid, and 3-phosphoglyceric acid) dominated the metabolome of G48 and G52, whereas several amines, including putrescine, allantoin, and triethanolamine, as well as asparagine and fumaric acid were the major components of the metabolomes of G58, G1710, and IR64 (Supplementary Table 1).

\section{Genotype-specific differences under control conditions}

A comparison of the five lines under control conditions indicated that the abundance of 70 of the 84 identified metabolites differed significantly among the lines (Table 3 ). Specifically, 51, 32, 13, 21, and 17 metabolites were most abundant in G1710, G58, IR64, G52, and G45, respectively. Eleven metabolites comprising two amines (allantoin and triethanolamine), four AAs (asparagine, L-lysine, serine, and leucine), four sugars (fructose, glucose, fructofuranose, and 2-o-glycerol-beta-d-galactopyranoside), and fumaric acid were generally detected at higher levels in G1710, G58, and IR64 than in the other two lines, especially for allantoin which almost ninefold higher. Moreover, compared with G45 and G52 levels, the average AA content was higher in G1710, G58, and IR64.

\section{Most amino acid and sugar levels increased in response to salinity, while lower levels were observed in most tolerant line}

Under salt stress conditions, the levels of 16 in 19 identified AAs varied significantly among lines (Supplementary Table 2). Four AAs (proline, phenylalanine, leucine, and isoleucine) were consistently detected at the highest 
Table 3 The relative content of metabolites in five rice lines under control condition

\begin{tabular}{|c|c|c|c|c|c|}
\hline & G45 & G52 & G1710 & G58 & IR64 \\
\hline \multicolumn{6}{|l|}{ Amino acids } \\
\hline Alanine & $39.4 \mathrm{~b}$ & $46.4 b$ & $60.2 \mathrm{a}$ & $59.9 \mathrm{a}$ & $48.4 \mathrm{~b}$ \\
\hline Asparagine & $20.7 b$ & $23.0 \mathrm{~b}$ & $48.7 \mathrm{a}$ & $48.5 \mathrm{a}$ & $46.1 \mathrm{a}$ \\
\hline Aspartic acid & $70.7 \mathrm{~b}$ & $61.9 \mathrm{~b}$ & $93.9 \mathrm{a}$ & $61.0 \mathrm{~b}$ & $68.6 \mathrm{~b}$ \\
\hline Butyric acid-4-amino & $47.5 \mathrm{a}$ & $48.1 \mathrm{a}$ & $45.2 \mathrm{a}$ & $54.7 \mathrm{a}$ & $48.2 \mathrm{a}$ \\
\hline Glutamic acid & $67.8 \mathrm{a}$ & $70.7 \mathrm{a}$ & $87.0 \mathrm{a}$ & $70.3 \mathrm{a}$ & $69.3 \mathrm{a}$ \\
\hline Glutamine & $13.2 \mathrm{c}$ & $15.1 \mathrm{c}$ & $33.4 \mathrm{a}$ & $27.6 \mathrm{a}$ & $20.6 b$ \\
\hline Glycine & $22.0 \mathrm{~cd}$ & $17.5 \mathrm{~d}$ & $41.9 \mathrm{a}$ & $31.3 \mathrm{~b}$ & $28.7 \mathrm{cb}$ \\
\hline Homoserine & $19.7 \mathrm{c}$ & $28.8 \mathrm{~b}$ & $47.8 \mathrm{a}$ & $37.1 \mathrm{~b}$ & $31.7 \mathrm{~b}$ \\
\hline Isoleucine & $18.3 \mathrm{a}$ & $18.6 \mathrm{a}$ & $24.0 \mathrm{a}$ & $22.3 \mathrm{a}$ & $20.0 \mathrm{a}$ \\
\hline Leucine & $18.8 \mathrm{a}$ & $22.3 \mathrm{a}$ & $25.2 \mathrm{a}$ & $25.7 \mathrm{a}$ & $23.7 \mathrm{a}$ \\
\hline L-Lysine & $15.8 \mathrm{c}$ & $17.7 \mathrm{~b}$ & $22.4 \mathrm{a}$ & $22.6 \mathrm{a}$ & $18.8 \mathrm{~b}$ \\
\hline Phenylalanine & $32.6 \mathrm{a}$ & $27.2 \mathrm{~b}$ & $22.1 \mathrm{c}$ & $22.1 \mathrm{c}$ & $19.0 \mathrm{c}$ \\
\hline Proline & $15.5 b$ & $19.9 \mathrm{ba}$ & $23.8 \mathrm{a}$ & $22.8 \mathrm{a}$ & $20.2 \mathrm{ba}$ \\
\hline Serine & $26.2 \mathrm{c}$ & $31.1 \mathrm{cb}$ & $62.5 \mathrm{a}$ & $54.7 \mathrm{a}$ & $40.3 b$ \\
\hline Threonine & $27.4 \mathrm{c}$ & $31.0 \mathrm{c}$ & $51.9 \mathrm{a}$ & $45.8 \mathrm{ba}$ & $37.4 \mathrm{bc}$ \\
\hline Tryptophan & $15.7 \mathrm{~b}$ & $16.9 \mathrm{~b}$ & $16.0 \mathrm{~b}$ & $34.9 \mathrm{a}$ & $16.2 b$ \\
\hline Tyramine & $27.2 \mathrm{cb}$ & $39.9 \mathrm{a}$ & $30.7 \mathrm{~b}$ & $28.4 \mathrm{cb}$ & $22.1 \mathrm{c}$ \\
\hline Tyrosine & $24.6 \mathrm{a}$ & $23.3 \mathrm{a}$ & $27.4 \mathrm{a}$ & $24.0 \mathrm{a}$ & $21.8 \mathrm{a}$ \\
\hline Valine & $11.9 \mathrm{a}$ & $12.8 \mathrm{a}$ & $17.5 \mathrm{a}$ & $15.8 \mathrm{a}$ & $15.2 \mathrm{a}$ \\
\hline \multicolumn{6}{|l|}{ Sugars } \\
\hline 2-O-glycerol-beta-d-galactopyranoside & $18.4 \mathrm{~b}$ & $32.6 \mathrm{a}$ & $31.9 \mathrm{a}$ & $28.9 \mathrm{a}$ & $27.4 \mathrm{a}$ \\
\hline Arabinose & $28.7 \mathrm{a}$ & $31.4 \mathrm{a}$ & $34.9 \mathrm{a}$ & $36.3 \mathrm{a}$ & $31.9 \mathrm{a}$ \\
\hline Cellobiose & $50.9 \mathrm{a}$ & $47.2 \mathrm{a}$ & $59.3 \mathrm{a}$ & $53.5 \mathrm{a}$ & $57.5 \mathrm{a}$ \\
\hline Erythritol & $17.7 \mathrm{~b}$ & $16.0 \mathrm{~b}$ & $31.4 \mathrm{a}$ & $22.5 b$ & $17.3 b$ \\
\hline Fructofuranose & $12.8 \mathrm{c}$ & $13.6 \mathrm{c}$ & $17.5 b$ & $30.5 \mathrm{a}$ & $16.8 \mathrm{~b}$ \\
\hline Fructose & $13.5 \mathrm{c}$ & $14.7 \mathrm{c}$ & $18.3 b$ & $30.3 \mathrm{a}$ & $17.6 \mathrm{~b}$ \\
\hline Fructose-6-P & $48.2 \mathrm{ba}$ & $42.8 b$ & $51.9 \mathrm{a}$ & $41.5 b$ & $42.3 b$ \\
\hline Galactose & $55.5 \mathrm{a}$ & $59.9 \mathrm{a}$ & $57.6 \mathrm{a}$ & $52.5 \mathrm{a}$ & $49.8 \mathrm{a}$ \\
\hline Glucose & $31.1 \mathrm{c}$ & $29.8 \mathrm{c}$ & $40.0 \mathrm{~b}$ & $59.3 \mathrm{a}$ & $40.5 b$ \\
\hline Glucose-6-P & $81.3 \mathrm{a}$ & $75.6 b$ & $87.3 \mathrm{a}$ & $79.7 \mathrm{a}$ & $72.2 b$ \\
\hline Glycerol & $34.9 \mathrm{a}$ & $33.2 \mathrm{a}$ & $41.9 \mathrm{a}$ & $35.2 \mathrm{a}$ & $33.5 \mathrm{a}$ \\
\hline Inositol & $24.8 \mathrm{a}$ & $23.0 \mathrm{a}$ & $24.9 \mathrm{a}$ & $21.1 \mathrm{a}$ & $21.6 \mathrm{a}$ \\
\hline Lactose & $48.0 \mathrm{bc}$ & $48.9 \mathrm{bc}$ & $59.8 \mathrm{a}$ & $45.0 \mathrm{c}$ & $54.3 \mathrm{ba}$ \\
\hline Maltose & $40.3 \mathrm{ba}$ & $33.2 \mathrm{~b}$ & $43.9 \mathrm{a}$ & $42.1 \mathrm{ba}$ & $36.4 b$ \\
\hline Mannose & $17.1 \mathrm{bc}$ & $11.6 \mathrm{c}$ & $16.6 \mathrm{bc}$ & $26.8 \mathrm{a}$ & $21.5 \mathrm{ba}$ \\
\hline Melezitos & $20.3 a$ & $22.3 \mathrm{a}$ & $27.2 \mathrm{a}$ & $24.5 \mathrm{a}$ & $20.6 a$ \\
\hline Raffinose & $15.2 \mathrm{a}$ & $15.3 \mathrm{a}$ & $7.9 \mathrm{~b}$ & $9.4 b$ & $10.7 \mathrm{~b}$ \\
\hline Salicylic acid-glucopyranoside & $16.8 \mathrm{c}$ & $22.1 \mathrm{~b}$ & $16.3 \mathrm{c}$ & $28.4 \mathrm{a}$ & $14.0 \mathrm{c}$ \\
\hline Sorbitol & $17.9 \mathrm{~b}$ & $19.1 \mathrm{~b}$ & $25.3 \mathrm{a}$ & $15.7 b$ & $16.1 \mathrm{~b}$ \\
\hline Sucrose & $24.7 \mathrm{a}$ & $27.1 \mathrm{a}$ & $28.7 \mathrm{a}$ & $25.9 \mathrm{a}$ & $26.2 \mathrm{a}$ \\
\hline Trehalose & $22.0 \mathrm{~b}$ & $23.2 b$ & $30.2 \mathrm{a}$ & $21.8 b$ & $24.1 \mathrm{~b}$ \\
\hline Xylitol & $16.4 \mathrm{~b}$ & $20.0 \mathrm{~b}$ & $33.2 \mathrm{a}$ & $24.6 \mathrm{~b}$ & $22.8 \mathrm{~b}$ \\
\hline Xylose & $15.9 \mathrm{a}$ & $13.8 \mathrm{a}$ & $15.2 \mathrm{a}$ & $13.6 \mathrm{a}$ & $9.6 \mathrm{~b}$ \\
\hline \multicolumn{6}{|l|}{ Organic acids } \\
\hline 2-Ketoglutaric acid & $48.7 \mathrm{a}$ & $48.9 \mathrm{a}$ & $41.1 \mathrm{a}$ & $41.6 \mathrm{a}$ & $41.7 \mathrm{a}$ \\
\hline Acetic acid & $35.5 \mathrm{a}$ & $28.8 \mathrm{~b}$ & $30.4 b$ & $21.3 \mathrm{c}$ & $21.9 \mathrm{c}$ \\
\hline Beta-d-glucopyranuronic acid & $20.3 \mathrm{a}$ & $27.8 \mathrm{a}$ & $24.9 \mathrm{a}$ & $25.9 \mathrm{a}$ & $21.7 \mathrm{a}$ \\
\hline Bistrimethylsilyl pyruvic acid & $32.7 \mathrm{~b}$ & $31.6 \mathrm{~b}$ & $26.9 \mathrm{c}$ & $38.1 \mathrm{a}$ & $25.5 \mathrm{c}$ \\
\hline Cinnamic acid-4-hydroxy & $31.5 \mathrm{a}$ & $30.1 \mathrm{a}$ & $35.3 \mathrm{a}$ & $37.1 \mathrm{a}$ & $32.7 \mathrm{a}$ \\
\hline Citric acid & $38.8 b$ & $46.2 \mathrm{a}$ & $38.0 \mathrm{cb}$ & $31.5 \mathrm{cb}$ & $30.7 \mathrm{c}$ \\
\hline
\end{tabular}


Table 3 (continued)

\begin{tabular}{|c|c|c|c|c|c|}
\hline & G45 & G52 & G1710 & G58 & IR64 \\
\hline Fumaric acid & $24.2 \mathrm{c}$ & $37.4 \mathrm{~b}$ & $61.3 \mathrm{a}$ & $44.9 b$ & $41.0 \mathrm{~b}$ \\
\hline Galactonic acid & $23.9 \mathrm{a}$ & $31.6 \mathrm{a}$ & $25.2 \mathrm{a}$ & $25.5 \mathrm{a}$ & $25.8 \mathrm{a}$ \\
\hline Gluconic acid & $27.7 \mathrm{a}$ & $31.0 \mathrm{a}$ & $18.7 \mathrm{~b}$ & $13.3 \mathrm{~b}$ & $15.9 \mathrm{~b}$ \\
\hline Glyceric acid & $52.3 \mathrm{bc}$ & $70.3 \mathrm{a}$ & $55.8 \mathrm{ba}$ & $38.2 \mathrm{c}$ & $47.0 \mathrm{bc}$ \\
\hline Glyceric acid-3-p & $54.3 \mathrm{ba}$ & $42.2 \mathrm{bc}$ & $61.4 \mathrm{a}$ & $15.4 d$ & $29.8 \mathrm{c}$ \\
\hline Isocitric acid & $6.6 \mathrm{c}$ & $23.7 \mathrm{~b}$ & $4.6 \mathrm{c}$ & $47.2 \mathrm{a}$ & $10.9 \mathrm{c}$ \\
\hline Malic acid & $59.8 \mathrm{~b}$ & $83.2 \mathrm{a}$ & $51.1 \mathrm{~b}$ & $46.8 b$ & $53.9 \mathrm{~b}$ \\
\hline Malonic acid & $44.3 \mathrm{c}$ & $32.3 \mathrm{~d}$ & $74.3 \mathrm{a}$ & $30.9 \mathrm{~d}$ & $55.9 \mathrm{~b}$ \\
\hline Octadecanoic acid & $35.0 \mathrm{a}$ & $29.9 \mathrm{a}$ & $31.7 \mathrm{a}$ & $30.2 \mathrm{a}$ & $34.3 \mathrm{a}$ \\
\hline Oleic acid & $50.2 \mathrm{a}$ & $28.2 \mathrm{c}$ & $31.7 \mathrm{c}$ & $42.7 b$ & $30.2 \mathrm{c}$ \\
\hline Oxalic acid & $81.9 \mathrm{a}$ & $62.8 b$ & $75.0 \mathrm{a}$ & $23.6 \mathrm{c}$ & $72.7 \mathrm{a}$ \\
\hline Phosphate & $48.0 \mathrm{a}$ & $41.4 b$ & $40.9 \mathrm{cb}$ & $35.1 \mathrm{c}$ & $38.4 \mathrm{cb}$ \\
\hline Pipecolic acid & $16.4 \mathrm{~b}$ & $20.1 b$ & $34.8 \mathrm{a}$ & $30.7 \mathrm{a}$ & $22.7 b$ \\
\hline Propanoic acid & $40.8 \mathrm{a}$ & $30.9 b c$ & $33.6 \mathrm{ba}$ & $28.8 \mathrm{bc}$ & $22.2 \mathrm{c}$ \\
\hline Quinic acid & $63.9 \mathrm{a}$ & $64.3 \mathrm{a}$ & $52.6 \mathrm{a}$ & $34.7 \mathrm{~b}$ & $49.0 \mathrm{ba}$ \\
\hline Saccharic acid & $68.3 \mathrm{a}$ & $58.0 \mathrm{a}$ & $67.6 \mathrm{a}$ & $43.3 b$ & $64.0 \mathrm{a}$ \\
\hline Succinic acid & $52.0 \mathrm{a}$ & $74.2 \mathrm{a}$ & $68.6 \mathrm{a}$ & $69.8 \mathrm{a}$ & $60.4 \mathrm{a}$ \\
\hline Threonic acid & $19.9 \mathrm{c}$ & $45.1 \mathrm{a}$ & $30.8 \mathrm{~b}$ & $17.7 \mathrm{c}$ & $22.0 \mathrm{c}$ \\
\hline \multicolumn{6}{|l|}{ Other small molecular components (SMC) } \\
\hline Allantoin & $11.7 \mathrm{~d}$ & $10.7 \mathrm{~d}$ & $63.5 b$ & $188.4 \mathrm{a}$ & $43.0 \mathrm{c}$ \\
\hline 2,4,6-Tri-tert-butylbenzenethiol & $36.6 \mathrm{a}$ & $32.6 \mathrm{a}$ & $36.4 \mathrm{a}$ & $35.2 \mathrm{a}$ & $37.4 \mathrm{a}$ \\
\hline Threonic acid-1,4-lactone & $17.7 \mathrm{~b}$ & $34.7 \mathrm{a}$ & $28.1 \mathrm{a}$ & $20.0 \mathrm{~b}$ & $14.5 b$ \\
\hline Monooctadecanoylglycerol & $37.7 \mathrm{a}$ & $34.4 \mathrm{a}$ & $33.9 \mathrm{a}$ & $33.8 \mathrm{a}$ & $38.4 \mathrm{a}$ \\
\hline 2-Methyl-1,3-butanediol & $21.0 \mathrm{~b}$ & $26.3 \mathrm{a}$ & $29.7 \mathrm{a}$ & $24.9 \mathrm{ba}$ & $23.2 \mathrm{ba}$ \\
\hline Ethylene glycol & $15.8 \mathrm{~b}$ & $29.8 \mathrm{a}$ & $27.1 \mathrm{a}$ & $16.2 \mathrm{~b}$ & $21.1 \mathrm{~b}$ \\
\hline 1-Monohexadecanoylglycerol & $39.8 \mathrm{a}$ & $34.5 b$ & $33.2 b$ & $33.4 \mathrm{~b}$ & $37.2 \mathrm{ba}$ \\
\hline Ethanolamine & $30.0 \mathrm{a}$ & $27.5 \mathrm{a}$ & $27.1 \mathrm{a}$ & $29.5 \mathrm{a}$ & $28.6 \mathrm{a}$ \\
\hline Triethanolamine & $12.5 \mathrm{c}$ & $20.9 b$ & $37.5 \mathrm{a}$ & $38.6 \mathrm{a}$ & $33.4 \mathrm{a}$ \\
\hline Putrescine & $10.5 \mathrm{c}$ & $17.3 \mathrm{c}$ & $26.2 b$ & $44.9 \mathrm{a}$ & $15.2 \mathrm{c}$ \\
\hline 2-Aminoethanol & $41.7 \mathrm{~b}$ & $38.3 b$ & $52.7 \mathrm{a}$ & $52.1 \mathrm{a}$ & $40.9 b$ \\
\hline Tryptamine-5-hydroxy & $163.0 \mathrm{~b}$ & $46.4 \mathrm{c}$ & $146.5 b$ & $279.2 \mathrm{a}$ & $32.6 \mathrm{c}$ \\
\hline Nicotianamine & $24.9 \mathrm{a}$ & $20.1 b$ & $23.9 \mathrm{a}$ & $18.0 \mathrm{~b}$ & $17.6 \mathrm{~b}$ \\
\hline Benzoic acid trimethylsilyl ester & $28.5 \mathrm{a}$ & $25.5 \mathrm{a}$ & $23.9 \mathrm{a}$ & $25.9 \mathrm{a}$ & $22.7 \mathrm{a}$ \\
\hline Phosphoric acid monomethyl ester & $46.8 \mathrm{~b}$ & $43.4 \mathrm{~b}$ & $57.3 \mathrm{a}$ & $45.2 \mathrm{~b}$ & $38.2 b$ \\
\hline Glycerol-3-P & $27.7 \mathrm{~b}$ & $34.3 \mathrm{ba}$ & $46.2 \mathrm{a}$ & $33.7 \mathrm{ba}$ & $40.0 \mathrm{ba}$ \\
\hline Hexadecanoic acid & $34.2 \mathrm{a}$ & $30.4 \mathrm{a}$ & $31.3 \mathrm{a}$ & $28.8 \mathrm{a}$ & $33.3 \mathrm{a}$ \\
\hline Adenosine-5-monophosphate (AMP) & $41.2 \mathrm{a}$ & $41.3 \mathrm{a}$ & $44.2 \mathrm{a}$ & $38.8 \mathrm{a}$ & $33.5 \mathrm{a}$ \\
\hline
\end{tabular}

The alphabet "a- $d$ " indicates the metabolites levels in five rice lines were significantly different under control condition

levels among the identified AAs in all five lines. The average AA content was generally lower in G1710, G58, and IR64 than in the other two lines. For example, the average AA content was $162.7,172.6,188.8,297.2$, and 256.2 in G1710, G58, IR64, G45, and G52, respectively. Moreover, compared with the corresponding contents in G45 and G52, the proline, tyrosine, and valine levels were obviously lower in G1710, G58, and IR64. The lowest average AA level was observed in G1710, and the abundance of five AAs (glutamine, phenylalanine, valine, leucine, and isoleucine) was lowest in G1710.

Compared with the control levels, the abundance of most of the AAs increased in response to salt stress in all five lines (Supplementary Table 2). The most pronounced increases were observed in G45 and G52. The salt stress-induced average AAs levels increased by 13.4, 9.9, 4.3, 4.9, and 6.8 fold in G45, G52, G1710, G58, and IR64, respectively. Compared with the G45 and G52 


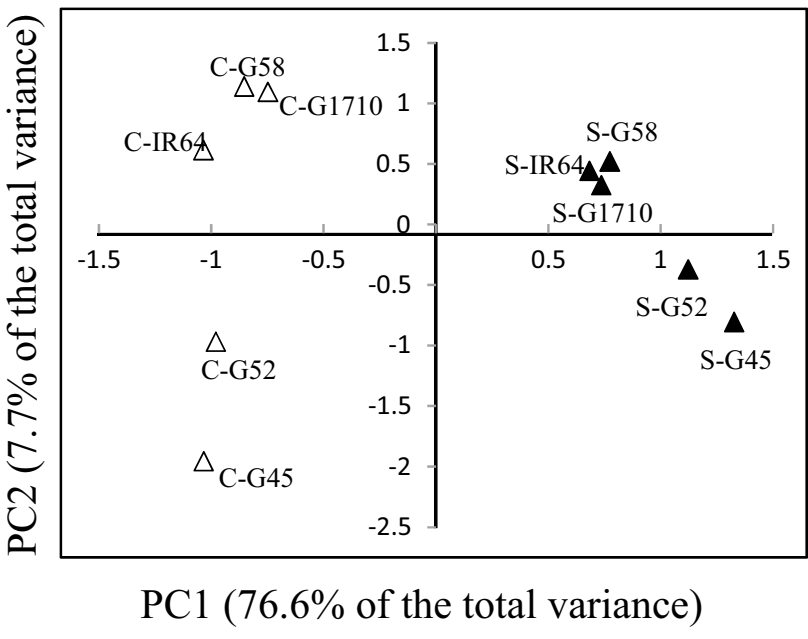

Fig. 1 The plot of the first two principal components of the metabolites in leaf of five rice lines under control (C) and salt (S) conditions at 3 days after salt treatment

contents, the change levels of seven AAs (glutamine, glycine, homoserine, serine, tyrosine, proline, and valine) were much lower in G1710, G58, and IR64. Additionally, G1710 had the most AAs with the lowest increases. Therefore, increases in AA contents were considered as an indicator of general stress and cell damage.

Of the 23 identified sugars and/or sugar derivatives, 19 were detected at varying levels among the lines (Supplementary Table 2). Galactose, sorbitol, arabinose, melezitose, maltose, lactose, and glucose were generally the most abundant in all five lines. Similar to the AA pattern, most sugars were detected at lower levels in G1710, G58, and IR64 than in G45 and G52. The average sugar contents were $72.8,86.1,76.9,127.8$, and 112.3 in G1710, G58, IR64, G45, and G52, respectively. The abundance of sorbitol, arabinose, galactose, melezitose, xylose, and fructofuranose were much lower in G1710, G58, and IR64 than in G5 and G52. Additionally, melezitose, sorbitol and raffinose contents were lowest in G1710.

Compared with the control levels, the abundance of 22 of the 23 sugars significantly increased in response to salt stress (Supplementary Table 2). The average contents of these 22 sugars increased by 1.4, 2.1, 2.0, 4.4, and 3.6 fold in G1710, G58, IR64, G45, and G52, respectively. Sorbitol, melezitose, and erythritol levels generally increased the most in the five lines. Compared with the corresponding changes in G45 and G52, the increases in fructofuranose, cellobiose, sorbitol, arabinose, fructose and glucose levels were considerably lower in G1710, G58, and IR64. Furthermore, the increases to sorbitol, melezitose, galactose, xylose, and maltose contents were lower in G1710 than in the other lines.

\section{Half of the organic acids and other small molecular components increased in abundance in response to salt stress}

Under saline condition, significant differences in abundance among the analyzed lines were observed for 19 of 24 identified OAs (Supplementary Table 2). In contrast to the sugar and AA contents, the differences in the average OA levels among lines were less obvious: 55.7, 53.3, 53.4, 73.2, and 60.7 in G1710, G58, IR64, G45, and G52, respectively. Among all metabolites, pipecolic acid was generally the most abundant in all lines. Compared with G45 and G52, lines G1710, G58, and IR64 generally had higher levels of five OAs (fumaric acid, quinic acid, 2-ketoglutaric acid, malic acid, and malonic acid), and lower levels of two OAs (threonic acid and galactonic acid). Among the five lines, G1710 had the highest glyceric acid and 3-phosphoglyceric acid contents, but the lowest isocitric acid content.

Compared with the corresponding levels under control conditions, the abundance of 12 of 24 OAs was significantly higher under saline conditions (Supplementary Table 2). The average contents of these 12 OAs increased by 0.9 , 1.4, 1.4, 3.1, and 1.5 fold in G1710, G58, IR64, G45, and G52, respectively. Six OAs (pipecolic acid, threonic acid, gluconic acid, galactonic acid, acetic acid, and beta-d-glucopyranuronic acid) were generally associated with the highest increases and the OA contents increased less in G1710, G58, and IR64 than in G45 and G52. Moreover, the lowest increase in pipecolic acid contents and highest increase in 2-ketoglutaric acid levels were observed in G1710.

Of the 18 identified small molecular components, the abundance of 13 significantly differed among the five lines (Supplementary Table 2). Under saline conditions, allantoin levels were higher in G1710, G58, and IR64 than in the other two lines, whereas 2-aminoethanol, nicotianamine, and threonic acid-1,4-lactone exhibited the opposite pattern. Compared with the levels under control conditions, the average contents of the 13 small molecular components that varied among lines increased by $0.8,1.0,1.5,2.5$, and 1.8 fold in G1710, G58, IR64, G45, and G52, respectively (Supplementary Table 2). Thus, the changes in the abundance of these SMCs were smaller in the three lines exhibiting better growth under saline conditions. The average allantoin levels were twofold higher in G1710, G58, and IR64 than in G45 and G52.

\section{Discussion}

Characterizing the physiological and metabolic basis for salt tolerance will be beneficial for rice breeders. Metabolomics research is useful for investigating the biological responses of plants to various stresses because it can be applied to 
identify diverse compounds, such as the by-products of stress-induced metabolism, stress-related signal transduction molecules, and molecules associated with plant acclimation to stress conditions (Shulaev et al. 2008; Anwar et al. 2016).

In this study, we analyzed four introgression lines and the recurrent parent, IR64, to investigate rice early metabolic responses to saline conditions before stress-induced symptoms are easily observable. Using plants with a similar genetic background as research materials can decrease the influence of complex genetic backgrounds on the results interpretation. We observed that G1710, G58, and IR64 grew better than G45 and G52 over an 8-day salt treatment, as evidenced by the higher SHT, SDW, and RDW values under saline conditions. The observed lower $\mathrm{Na}^{+}$content and higher $\mathrm{K}^{+} / \mathrm{Na}^{+}$ratio in G58, G1710, and IR64 may at least partially explain why these plants grew better than the G45 and G52 plants (Table 1). Of all analyzed lines, G1710 grew best and was the least adversely affected by saline conditions.

We were especially interested in the metabolic differences among the analyzed lines. In response to stress, the metabolic homeostasis of plants is disrupted and the metabolic pathways adjust to the adverse conditions in a process that is usually referred to as acclimation (Anwar et al. 2016). For example, several studies revealed that changes to OA and AA contents as well as sugar metabolism are part of the general response toward stress (Anwar et al. 2016; Sharma et al. 2016; El-Shintinawy and El-Shourbagy 2001). At present study, it's found, under control conditions, lines G1710, G58, and IR64 had higher average AA contents than those in G45 and G52, which suggested the higher abundance of some compounds with protective functions against the osmotic imbalance prior salt stress were part of the intrinsic differences between the salt-sensitive and salt-tolerant lines. The result was consistent with previous reports (Hasegawa et al. 2000; Gong et al. 2005). Additionally, G1710 had more metabolites at the highest levels than the other analyzed lines, which may have contributed to its stress tolerance.

The principal component analysis revealed that the salinity-induced metabolite contents and plants with varying tolerance to salt stress were clearly separated by the first two principal components, implying the identified metabolites were significantly correlated with plant growth (Fig. 1). As observed in previous studies (Zhao et al. 2014; Gray and Heath 2005; Hannah et al. 2006; Kaplan et al. 2004), metabolic activities are largely modified in plants exposed to stresses (Fig. 1 and Supplementary Table 1). Loading analysis indicated that allantoin, putrescine, and triethanolamine were the major contributors to the metabolomes of G58, G1710, and IR64 (Supplementary Table 1), which is partly consistent with the ANOVA results (Supplementary Table 2). Earlier investigations confirmed allantoin contents increase in response to diverse stress conditions (Lescano et al. 2016; Watanabe et al. 2014; Wang et al. 2016; Nourimand and Todd 2016; Rose et al. 2012; Irani and Todd 2016), and allantoin protects plants from abiotic stresses by minimizing oxidative damage (Nourimand and Todd 2016) or activating abscisic acid metabolism (Watanabe et al. 2014). The result indicated the allantoin accumulation is essential for salt stress tolerance. Polyamines function as antioxidants to protect plants from oxidative damage and maintain cellular homeostasis (Rodriguez-Kessler et al. 2006; Sharma et al. 2016). Thus, the higher contents of allantoin, putrescine, and triethanolamine were likely among the main reasons for the better growth of G1710, G58, and IR64 under control and/or saline conditions.

Moreover, the result showed the same metabolic pathways were regulated by salt stress in the sensitive and tolerant lines, the main difference between the two groups was there were more increase in metabolites in G45 and G52 (i.e., greater inhibited growth under saline conditions) than in G58, G1710, and IR64 (Table 1). For example, the abundance of proline, which is a well-known component of osmotic stress responses, increased by $60.9,32.2,17.8,11.7$, and 22.0 fold in G45, G52, G58, G1710, and IR64, respectively. These results were consistent with those of previous studies that determined that proline levels increase in both salt-tolerant and salt-sensitive species (Taji et al. 2004; Gong et al. 2005; Dittami et al. 2012; Verbruggen and Hermans 2008). Thus, the results confirmed the opinion that the main difference in metabolic response to stress between sensitive and tolerant species was quantitative (Lugan et al. 2010).

Interestingly, the data presented herein showed that more metabolites accumulated in the salt-sensitive lines than in the salt-tolerant lines, which contradicts the findings of earlier investigations of salinity-stressed plants (Zhao et al. 2014; Wang et al. 2016; Zuther et al. 2007; Gong et al. 2005). The contradictory results obtained in the current study may have been due to differences in the sampling times. Previous studies mostly focused on plants at 7 or more days after salt treatments when obvious stress-induced symptoms were observable. In contrast, we analyzed samples at 3 days after salt treatments when symptoms were essentially undetectable. These results imply the salt-sensitive lines have a more pronounced response than the salt-tolerant lines during the early time-points after stress treatments. A similar observation was reported for a time-series study on salt tolerance (Zhao et al. 2014). The accumulation of OAs, AAs, and sugars, which are the primary metabolites, is reportedly responsible for osmotic readjustments as these compounds are the direct markers of photosynthetic dysfunction (Anwar et al. 2016) and indicators of general stress and cell damage (Widodo et al. 2009). We consider the accumulation of these metabolites in response to salt stress is an adaptive response of the plants to saline conditions. Lines G45 and G52 appear to have been more sensitive to salt stress than the 
other lines during the early stages of the salt treatment, and responded to the adverse conditions partly by accumulating metabolites.

Several metabolites with common changes in abundance among all five lines under stress conditions were considered as fundamental components for adaptation. Specifically, the abundance of 13 metabolites comprising four AAs (proline, phenylalanine, leucine, and isoleucine), seven sugars (galactose, sorbitol, arabinose, maltose, lactose, glucose, and melezitose), pipecolic acid, and allantoin generally increased considerably in the five analyzed lines in response to salinity stress. These results suggest the metabolites may be as fundamental as proline for the osmotic stress tolerance that ensures plants can grow under saline conditions. Among them, sorbitol, melezitose, and pipecolic acid levels were detected at high levels in the five lines. It's reported pipecolic acid is correlated with an abnormal plant physiological condition or with some disturbance in AA or protein metabolism (Palfi and Dezsi 1968). Sorbitol is one of the main forms of photosynthetic and translocated carbon, and plant photosynthetic activities significantly decrease as the sorbitol concentration increases (Gao et al. 2014). More detail works on functions of target metabolites in stress-tolerance should be conducted in future research.

\section{Conclusion}

The salt tolerance of four rice introgression lines and a recurrent parent, IR64, were evaluated at the seedling stage. Lines G58, IR64, and especially G1710 were substantially more tolerant to salt stress than G52 and G48. The differences in the metabolite contents of five lines under control and saline conditions were assessed by GC-MS. The results suggested that increased allantoin, putrescine, and triethanolamine contents under control and/or salt conditions were related to the better performance of G1710, G58, and IR64. Significantly higher levels of AAs in G1710, G58, and IR64 may indicate these AAs have an intrinsic role in the salt tolerance of rice plants. Moreover, compared with proline contents, sorbitol, melezitose, and pipecolic acid levels increased more in response to stress, with relatively high concentrations among the five lines under saline conditions, which indicated their importance for rice responses to salt stress. Furthermore, the same metabolic pathways are likely regulated by salt stress in the sensitive and tolerant species, with the main difference being quantitative.

Acknowledgements This research was funded by the National Natural Science Foundation of China (31471429), the National Key Technology Support Program of China (2015BAD02B01), the Bill \& Melinda Gates Foundation Project (OPP51587), the CAAS Innovative Team Award to J.L. Xu's team. We thank Liwen Bianji, Edanz Editing China (www.liwenbianji.cn/ac) for editing the English text of a draft of this manuscript.

\section{Compliance with ethical standards}

Conflict of interest The authors declare no conflict of interest.

Open Access This article is licensed under a Creative Commons Attribution 4.0 International License, which permits use, sharing, adaptation, distribution and reproduction in any medium or format, as long as you give appropriate credit to the original author(s) and the source, provide a link to the Creative Commons licence, and indicate if changes were made. The images or other third party material in this article are included in the article's Creative Commons licence, unless indicated otherwise in a credit line to the material. If material is not included in the article's Creative Commons licence and your intended use is not permitted by statutory regulation or exceeds the permitted use, you will need to obtain permission directly from the copyright holder. To view a copy of this licence, visit http://creativecommons.org/licenses/by/4.0/.

\section{References}

Anwar K, Lakra N, Singla-Pareek SL, Pareek A (2016) Investigating abiotic stress response machinery in plants: the metabolomic approach. In: Dagar J, Sharma P, Sharma D, Singh A (eds) Innovative saline agriculture. Springer, New Delhi, pp 303-319

Banerjee A, Ghosh P, Roychoudhury A (2019) Salt acclimation differentially regulates the metabolites commonly involved in stress tolerance and aroma synthesis in indica rice cultivars. Plant Growth Regul 88:87-97

Bowne JB, Erwin TA, Juttner J, Schnurbusch T, Langridge P, Bacic A, Roessner U (2012) Drought responses of leaf tissues from wheat cultivars of differing drought tolerance at the metabolite level. Mol Plant 5:418-429

Dittami SM, Gravot A, Goulitquer S, Rousvoal S, Peters AF, Bouchereau A, Boyen C, Tonon T (2012) Towards deciphering dynamic changes and evolutionary mechanisms involved in the adaptation to low salinities in Ectocarpus (brown algae). Plant J 71:366-377

El-Shintinawy F, El-Shourbagy MN (2001) Alleviation of changes in protein metabolism in NaCl-stressed wheat seedlings by Thiamine. Biol Plant 44:541-545

Gao S, Zheng ZB, Gu WH, Xie XJ, Huan L, Pan GH, Wang GC (2014) Photosystem I shows a higher tolerance to sorbitolinduced osmotic stress than photosystem II in the intertidal macro-algae Ulva prolifera (Chlorophyta). Physiol Plant $152: 380-388$

Garg AK, Kim JK, Owens TG, Ranwala AP, Choi YD, Kochian LV, Wu RJ (2002) Trehalose accumulation in rice plants confers high tolerance levels to different abiotic stresses. PNAS 99:15898-15903

Gong QQ, Li PH, Ma SS, Indu RS, Bohnert HJ (2005) Salinity stress adaptation competence in the extremophile Thellungiella halophila in comparison with its relative Arabidopsis thaliana. Plant $\mathrm{J}$ 44:826-839

Gray GR, Heath D (2005) A global reorganization of the metabolome in Arabidopsis during cold acclimation is revealed by metabolic fingerprinting. Physiol Plant 124:236-248

Groppa MD, Benavides MP (2008) Polyamines and abiotic stress: recent advances. Amino Acids 34:35-45 
Hannah MA, Wiese D, Freund S, Fiehn O, Heyer AG, Hincha DK (2006) Natural genetic variation of freezing tolerance in Arabidopsis. Plant Physiol 142:98-112

Hasegawa PM, Bressan RA, Zhu JK, Bohnert HJ (2000) Plant cellular and molecular responses to high salinity. Annu Rev Plant Biol 51:463-499

Irani S, Todd CD (2016) Ureide metabolism under abiotic stress in Arabidopsis thaliana. J Plant Physiol 199:87-95

Islam MR, Gregorio GB, Salam AM, Collard BCY, Singh RK, Hassan L (2012) Validation of Saltol linked markers and haplotype diversity on chromosome 1 of rice. Mol Plant Breed 3:103-114

Jahan N, Zhang Y, Lv Y, Song MQ, Zhao CY, Hu HT, CuiYT WZW, Yang SL, Zhang AP, Hu J, Ye GY, Qian Q, Gao ZY, Guo LB (2020) QTL analysis for rice salinity tolerance and fine mapping of a candidate locus $q S L 7$ for shoot length under salt stress. Plant Growth Regul 90:307-319

Kaplan F, Kopka J, Haskell DW, Zhao W, Schiller KC, Gatzke N, Sung DY, Guy CL (2004) Exploring the temperature-stress metabolome of Arabidopsis. Plant Physiol 136:4159-4168

Kim BG, Waadt R, Cheong YH, Pandey GK, Dominguez-Solis JR, Schultke S, Lee SC, Kudla J, Luan S (2007) The calcium sensor CBL10 mediates salt tolerance by regulating ion homeostasis in Arabidopsis. Plant J 52:473-484

Lescano CI, Martini C, González CA, Desimone M (2016) Allantoin accumulation mediated by allantoinase downregulation and transport by Ureide Permease 5 confers salt stress tolerance to Arabidopsis plants. Plant Mol Biol 91:581-595

Li ZK, Fu BY, Gao YM, Xu JL, Ali J, Lafitte HR, Jiang YZ, Rey JD, Vijayakumar CH, Maghirang R, Zheng TQ, Zhu LH (2005) Genome-wide introgression lines and their use in genetic and molecular dissection of complex phenotypes in rice (Oryza sativa $\mathrm{L}$.). Plant Mol Biol 59:33-52

Liu Y, Wang BX, Li J, Song ZQ, Lu BG, Chi M, Yang B, Liu JB, Lam YW, Li JX, Xu DY (2017) Salt-response analysis in two rice cultivars at seedling stage. Acta Physiol Plant 39:215. https ://doi.org/10.1007/s11738-017-2514-6

Lugan R, Niogret MF, Leport L, Guegan JP, Larher FR, Savoure A, Kopka J, Bouchereau A (2010) Metabolome and water homeostasis analysis of Thellungiella salsuginea suggests that dehydration tolerance is a key response to osmotic stress in this halophyte. Plant J 64:215-229

Ma XS, Xia H, Liu YH, Wei HB, Zheng XG, Song CZ, Chen L, Liu HY, Luo LJ (2016) Transcriptomic and metabolomic studies disclose key metabolism pathways contributing to wellmaintained photosynthesis under the drought and the consequent drought-tolerance in rice. Front Plant Sci. https://doi. org/10.3389/fpls.2016.01886

Munns R, Tester M (2008) Mechanisms of salinity tolerance. Ann Rev Plant Biol 59:651-681

Negrão S, Courtois B, Ahmadi N, Abreu I, Saibo N, Oliveira MM (2011) Recent updates on salinity stress in rice: from physiological to molecular responses. Crit Rev Plant Sci 30:329-377

Nemat Alla MM, Khedr AHA, Serag MM, Abu-Alnaga AZ, Nada RM (2012) Regulation of metabolomics in Atriplex halimus growth under salt and drought stress. Plant Growth Regul 67:281-304

Nourimand M, Todd CD (2016) Allantoin increases cadmium tolerance in Arabidopsis via activation of antioxidant mechanisms. Plant Cell Physiol 57:2485-2496

Nuccio M, Wu J, Mowers R, Zhou H, Meghji M, Primavesi LF et al (2015) Expression of trehalose-6-phosphate phosphatase in maize ears improves yield in well-watered and drought conditions. Nat Biotechnol 33:862-869
Okazaki Y, Saito K (2016) Integrated metabolomics and phytochemical genomics approaches for studies on rice. Giga Sci 5:11. https ://doi.org/10.1186/s13742-016-0116-7

Pal M, Szalai G, Janda T (2015) Speculation: polyamines are important in abiotic stress signaling. Plant Sci 237:16-23

Palfi G, Dezsi L (1968) Pipecolic acid as an indicator of abnormal protein metabolism in diseased plants. Plant Soil 29:285-291

Pearson GA, Bernstein L (1959) Salinity effects at several growth stages of rice. Agron J 51:654-657

Pearson GA, Ayers AD, Eberhard DL (1966) Relative salt tolerance of rice during germination and early seedling development. Soil Sci 102:151-156

Raval SS, Mahatma MK, Chakraborty K, Bishi SK, Singh AL, Rathod KJ, Jadav JK, Sanghani JM, Mandavia MK, Gajera HP, Golakiya BA (2018) Metabolomics of groundnut (Arachis hypogaea $\mathrm{L}$.) genotypes under varying temperature regimes. Plant Growth Regul 84:493-505

Rodriguez-Kessler M, Alpuche-Solis AG, Ruiz OA, Jimenez-Bremont JF (2006) Effect of salt stress on the regulation of maize (Zea mays L.) genes involved in polyamine biosynthesis. Plant Growth Regul 48:175-185

Rose MT, Rose TJ, Pariasca-Tanaka J, Yoshihashi T, Neuweger H, Goesmann A, Frei M, Wissuwa M (2012) Root metabolic response of rice (Oryza sativa L.) genotypes with contrasting tolerance to zinc deficiency and bicarbonate excess. Planta 236:959-973

Sharma PC, Prashat GR, Kumar A, Mann A (2016) Physiological and molecular insights into mechanisms for salt tolerance in plants. In: Dagar J, Sharma P, Sharma D, Singh A (eds) Innovative saline agriculture. Springer, New Delhi, pp 321-349

Shulaev V, Cortes D, Miller G, Mittler R (2008) Metabolomics for plant stress response. Physiol Plant 132:199-208

Singh M, Singh A, Prasad SM, Singh RK (2017) Regulation of plants metabolism in response to salt stress: an omics approach. Acta Physiol Plant 39:48. https://doi.org/10.1007/s11738-016-2345-x

Sun Y, Zang JP, Wang Y, Zhu LH, Fotokian M, Xu JL, Li ZK (2007) Mining favorable salt-tolerant QTL from rice germplasm using a backcrossing introgression line population. Acta Agron Sin 33:1611-1617

Taji T, Ohsumi C, Iuchi S, Seki M, Kasuga M, Kobayashi M, Shinozaki KY, Shinozaki K (2002) Important roles of drought-and cold-inducible genes for galactinol synthase in stress tolerance in Arabidopsis thaliana. Plant J 29:417-426

Taji T, Seki M, Satou M, Sakurai T, Kobayashi M, Ishiyama K, Narusaka Y, Narusaka M, Zhu JK, Shinozaki K (2004) Comparative genomics in salt tolerance between Arabidopsis and Arabidopsis-related halophyte salt cress using Arabidopsis microarray. Plant Physiol 135:1697-1709

Thomson MJ, Ocampo M, Egdane J, Rahman MA, Sajise AG, Adorada DL, Raiz E, Blumwald E, Seraj ZI, Singh RK, Gregorio GB, Ismail AM (2010) Characterizing the Saltol quantitative trait locus for salinity tolerance in rice. Rice 3:148-160

Verbruggen N, Hermans C (2008) Proline accumulation in plants: a review. Amino Acids 35:753-759

Walia H, Wilson C, Condamine P, Liu X, Ismail AM, Zeng LH, Wanamaker SI, Mandal J, Xu J, Cui XP, Close TJ (2005) Comparative transcriptional profiling of two contrasting rice genotypes under salinity stress during the vegetative growth stage. Plant Physiol 139:822-835

Wang WS, Zhao XQ, Li M, Huang LY, Xu JL, Zhang F, Cui YR, Fu BY, Li ZK (2016) Complex molecular mechanisms underlying seedling salt tolerance in rice revealed by comparative transcriptome and metabolomic profiling. J Exp Bot 67:405-419

Watanabe S, Matsumoto M, Hakomori Y, Takagi H, Shimada H, Sakamoto A (2014) The purine metabolite allantoin enhances 
abiotic stress tolerance through synergistic activation of abscisic acid metabolism. Plant Cell Environ 37:1022-1036

Widodo, Patterson JH, Newbigin ED, Tester M, Bacic A, Roessner U (2009) Metabolic responses to salt stress of barley (Hordeum vulgare L.) cultivars Sahara and Clipper, which differ in salinity tolerance. J Exp Bot 60:4089-4103

Yoshida S, Forna DA, Cock JH, Gomez KA (1976) Laboratory manual for physiological studies of rice. International Rice Research Institute, Los Banos, Philippines, pp 61-66

Zhao XQ, Wang WS, Zhang F, Deng JL, Li ZK, Fu BY (2014) Comparative metabolite profiling of two rice genotypes with contrasting salt stress tolerance at the seedling stage. PLoS ONE. https://doi.org/10.1371/journal.pone.0108020

Zhao XQ, Wang WS, Xie ZY, Gao YM, Wang CC, Rashid MM, Islam MR, Fu BY, Li ZK (2018) Comparative analysis of metabolite changes in two contrasting rice genotypes in response to low nitrogen stress. Crop J 6:464-474

Zuther E, Koehl K, Kopka J (2007) Comparative metabolome analysis of the salt response in breeding cultivars of rice. In: Jenks MA, Hasegawa PM, Jain SM (eds) Advances in molecular breeding toward drought and salt tolerance crops. Springer, Dordrecht, pp 285-315

Publisher's Note Springer Nature remains neutral with regard to jurisdictional claims in published maps and institutional affiliations. 